\title{
Transverse spin asymmetries at COMPASS: beyond Collins and Sivers effects
}

\author{
Bakur Parsamyan* \\ Dipartimento di Fisica Generale, Università di Torino, Torino, Italy \\ INFN, Sezione di Torino, Via P. Giuria 1, I-10125 Torino, Italy \\ E-mail: bakur.parsamyandcern.ch
}

One of the important objectives of the COMPASS experiment (SPS, CERN) [四] is the exploration of the transverse spin structure of the nucleon via spin dependent azimuthal asymmetries in single-hadron production in deep inelastic scattering of polarized leptons off transversely polarized targets. For this purpose a series of measurements were made in COMPASS, using 160 $\mathrm{GeV} / \mathrm{c}$ longitudinally polarized muon beam and transversely polarized ${ }^{6} \mathrm{LiD}$ (in 2002, 2003 and 2004) and $\mathrm{NH}_{3}$ (in 2007 and 2010) targets. In the past few years considerable theoretical interest and experimental efforts were focused on the study of Collins and Sivers transverse spin asymmetries. The experimental results obtained so far play an important role in the general understanding of the three-dimensional nature of the nucleon in terms of transverse momentum dependent parton distribution functions. In addition to these two measured leading-twist effects, the SIDIS cross-section includes six more target transverse spin dependent azimuthal asymmetries, which have their own well defined leading or higher-twist interpretation in terms of QCD parton model. COMPASS preliminary results for these six "beyond Collins and Sivers" asymmetries, obtained from transversely polarized deuteron and proton data have been presented at the previous conferences [] - [四]. In this review we focus on the results obtained with the last "proton-2010" data sample.

XXI International Workshop on Deep-Inelastic Scattering and Related Subject -DIS2013,

22-26 April 2013

Marseilles, France

\footnotetext{
* Speaker.
} 


\section{Introduction}

Measurement of the azimuthal asymmetries in SIDIS of leptons off polarized proton and deuteron targets is a powerful tool to study the spin structure of the nucleon. Schematic view of the SIDIS framework and some notations and definitions adopted in this letter ${ }^{1}$ such as system of axes, azimuthal angles, target polarization, etc. are presented in Fig. W. Here the target transverse polarization $\left(S_{T}\right)$ is defined relative to the virtual photon momentum direction (which is a most natural basis from the theory point of view) while, in experiment transverse polarization $\left(P_{T}\right)$ is defined relative to the beam (incoming lepton) direction. As it was demonstrated in [G]-[四] this difference, in particular, influences azimuthal distributions in the final state. After the appropriate conversions, the model-independent expression for the SIDIS cross-section for transversely (w.r.t. lepton beam) polarized target can be re-written in the following way [ [5]-[प]:

$$
\begin{aligned}
& \frac{d \sigma}{d x d y d z d P_{h T}^{2} d \varphi_{h} d \varphi_{S}}= \\
& {\left[\frac{\cos \theta}{1-\sin ^{2} \theta \sin ^{2} \varphi_{S}}\right]\left[\frac{\alpha}{x y Q^{2}} \frac{y^{2}}{2(1-\varepsilon)}\left(1+\frac{\gamma^{2}}{2 x}\right)\right]\left(F_{U U, T}+\varepsilon F_{U U, L}\right) \times}
\end{aligned}
$$

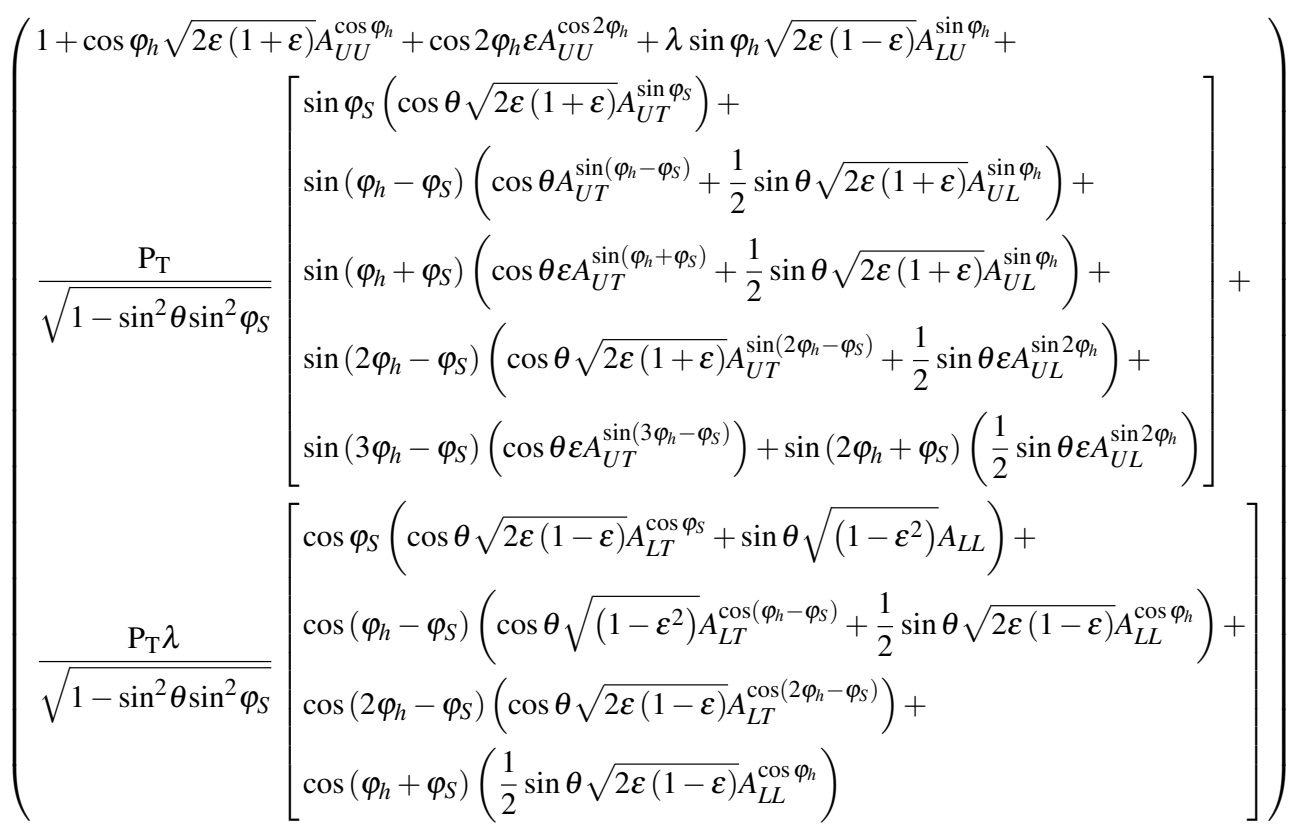

When compared, for instance, with the cross-section given in []]-[B]], in which the effects due to the $P_{T}$ to $S_{T}$ transition have been neglected, in this expression there are new $\sin \theta$-scaled terms and $\theta$-depending factors and two extra azimuthal modulations $\left(\sin \left(2 \varphi_{h}+\varphi_{S}\right)\right.$ and $\left.\cos \left(\varphi_{h}+\varphi_{S}\right)\right)$.

The equation $(\mathbb{L} \mathbb{C})$ counts in total eight: five Single-Spin (SSA) and three Double-Spin (DSA) target transverse polarization dependent asymmetries. Since $\sin \theta$ is a rather small quantity in COMPASS kinematics (see Fig. (D) the influence of the additional terms, represented by $\sin \theta$-scaled longitudinal-spin amplitudes and $\theta$-angle dependent factors, is sizable only in the case of $A_{L T}^{\cos \varphi_{S}}$ DSA, which, even taking into account the smallness of $\sin \theta$, is still sizably affected by a large $A_{L L}$ amplitude [ [B]. To correct the $A_{L T}^{\cos \varphi_{S}}$ asymmetry we have used the $A_{L L}$ values evaluated based on [团]). Corresponding $A_{L L}$ curves are shown in Fig.[ $]$ compared with the COMPASS data points [ [ 8$]$, demonstrating close agreement.

\footnotetext{
${ }^{1}$ The notations applied in this contribution are equivalent to those adopted in [四], [四], [四]-[四] and $\theta$ is the angle between $\gamma^{*}$-direction and initial lepton momenta (Fig.W)
} 
Other transverse amplitudes (including Collins and Sivers) are in much more favorable situation, since they either are not affected by any longitudinal amplitude $\left(A_{U T}^{\sin \left(3 \phi_{h}-\phi_{s}\right)}, A_{U T}^{\sin \left(\phi_{s}\right)}\right.$ and $\left.A_{L T}^{\cos \left(2 \phi_{h}-\phi_{s}\right)}\right)$, or are affected by $A_{U L}^{\sin \left(\phi_{h}\right)}, A_{U L}^{\sin \left(2 \phi_{h}\right)}$ or $A_{L L}^{\cos \left(\phi_{h}\right)}$ amplitudes which appear to be small at COMPASS with deuteron target [ए0] and are estimated to be of order of few percent with proton target (see, for instance, predictions for $A_{L L}^{\cos \left(\phi_{h}\right)}$ at COMPASS in [焑]). The effect of possible corrections has been studied on the example of $A_{L T}^{\cos \left(\phi_{h}-\phi_{s}\right)}$ asymmetry which was corrected for the $A_{L L}^{\cos \left(\phi_{h}\right)}$-contribution from [可]). It was demonstrated that the effect from longitudinal amplitude contribution is negligible. The eight target transverse spin dependent "raw" asymmetries are extracted simultaneously, using an unbinned maximum likelihood method and then are corrected for the $D$ depolarization factors ( $\varepsilon$-depending factors in equation (ㅍ. $\mathrm{d}$ ) standing in front of the amplitudes), dilution factor and target and beam (only DSAs) polarizations [[], []] . Obtained from COMPASS data

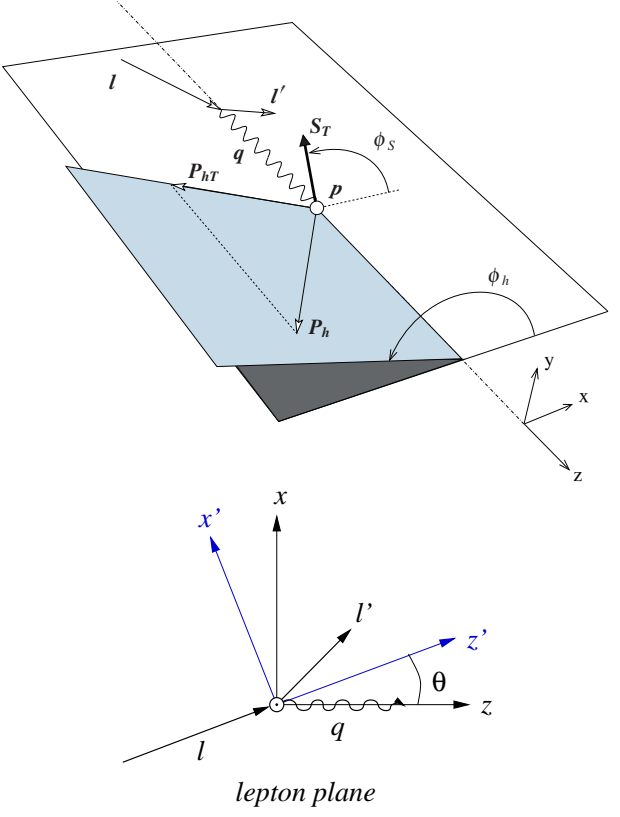

Figure 1: SIDIS framework (top) and scheme of $l p \rightarrow \gamma^{*} p$ transition (bottom) mean values of the $D$ factors corresponding to different asymmetries are presented in Fig.】.

In the QCD parton model approach four of the eight transverse spin asymmetries $\left(A_{U T}^{\sin \left(\phi_{h}+\phi_{S}\right)}\right.$, $A_{U T}^{\sin \left(\phi_{h}+\phi_{S}\right)}, A_{U T}^{\sin \left(3 \phi_{h}-\phi_{s}\right)}$ SSAs and $A_{L T}^{\cos \left(\phi_{h}-\phi_{s}\right)}$ DSA) have Leading Order (twist) (LO) interpretation and are described by the convolutions of the twist-two TMD PDFs and FFs, while the other four $\left(A_{U T}^{\sin \left(\phi_{s}\right)}\right.$ and $A_{U T}^{\sin \left(2 \phi_{h}-\phi_{s}\right)}$ SSAs and $A_{L T}^{\cos \left(\phi_{s}\right)}$ and $A_{L T}^{\cos \left(2 \phi_{h}-\phi_{s}\right)}$ DSAs), despite their higher-twist origin, however, can be represented as "Cahn kinematic corrections" to twist-two effects. These subleading amplitudes are suppressed with respect to the leading twist ones by $\sim M / Q$ (for details see: [目],[四],[四]-[目]). It can be shown that $\mathrm{LO} A_{U T}^{\sin \left(3 \phi_{h}-\phi_{s}\right)}$ (related to the $h_{1 T}^{q \perp}$ "pretzelosity" PDF) is expected to scale according to $\sim\left|\mathbf{P}_{\mathbf{h T}}{ }^{\mathbf{3}}\right|$ and thus is suppressed by $\sim\left|\mathbf{P}_{\mathbf{h T}}\right|^{\mathbf{2}}$ w.r.t $\sim\left|\mathbf{P}_{\mathbf{h T}}\right|$-scaled Collins, Sivers and $A_{L T}^{\cos \left(\phi_{h}-\phi_{s}\right)}$ LO amplitudes. Similarly, the other four asymmetries are suppressed by $\sim\left|\mathbf{P}_{\mathbf{h T}}\right|$. The $A_{L T}^{\cos \left(\phi_{h}-\phi_{s}\right)}$ amplitude (related to the $g_{1 T}^{q \perp}$ "worm gear" PDF) is of particular interest because it is the only transverse DSA expected to be sizable (LO, no suppression).

For Collins and Sivers effects, COMPASS has recently published results from 2010 proton data [[2], [ए3]]. In the next section we present the preliminary results for the other six asymmetries obtained from the same data sample.

\section{Data analysis and results}

The whole data selection and analysis procedure applied for the extraction of the six mentioned asymmetries from COMPASS 2010 proton data is identical to those applied for the already published Collins and Sivers asymmetries. The detailed description of COMPASS spectrometer

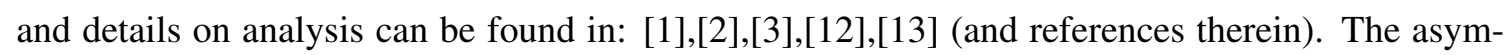


metries extracted as functions of $x, z$ and $P_{h T}$ for positive and negative hadrons are shown in Fig.[1]. The systematic uncertainties for each asymmetry have been estimated separately for positive and negative hadrons and are given by the bands. According to the preliminary observations, there is an evidence of non-zero LO $A_{L T}^{\cos \left(\phi_{h}-\phi_{s}\right)}$ DSA and sub-leading $A_{U T}^{\sin \left(\phi_{s}\right)}$ SSA, while the other four "beyond Collins and Sivers" amplitudes are found to be compatible with zero within the statistical accuracy. It has to be mentioned that similar behavior for both non-zero amplitudes and for zero-compatible amplitudes was already preliminary reported also by the HERMES collaboration.

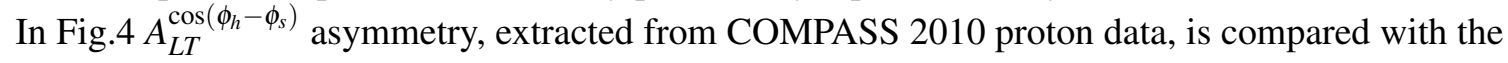

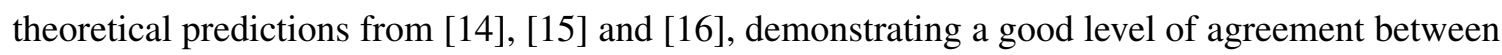
theory and measurement within the given statistical accuracy.

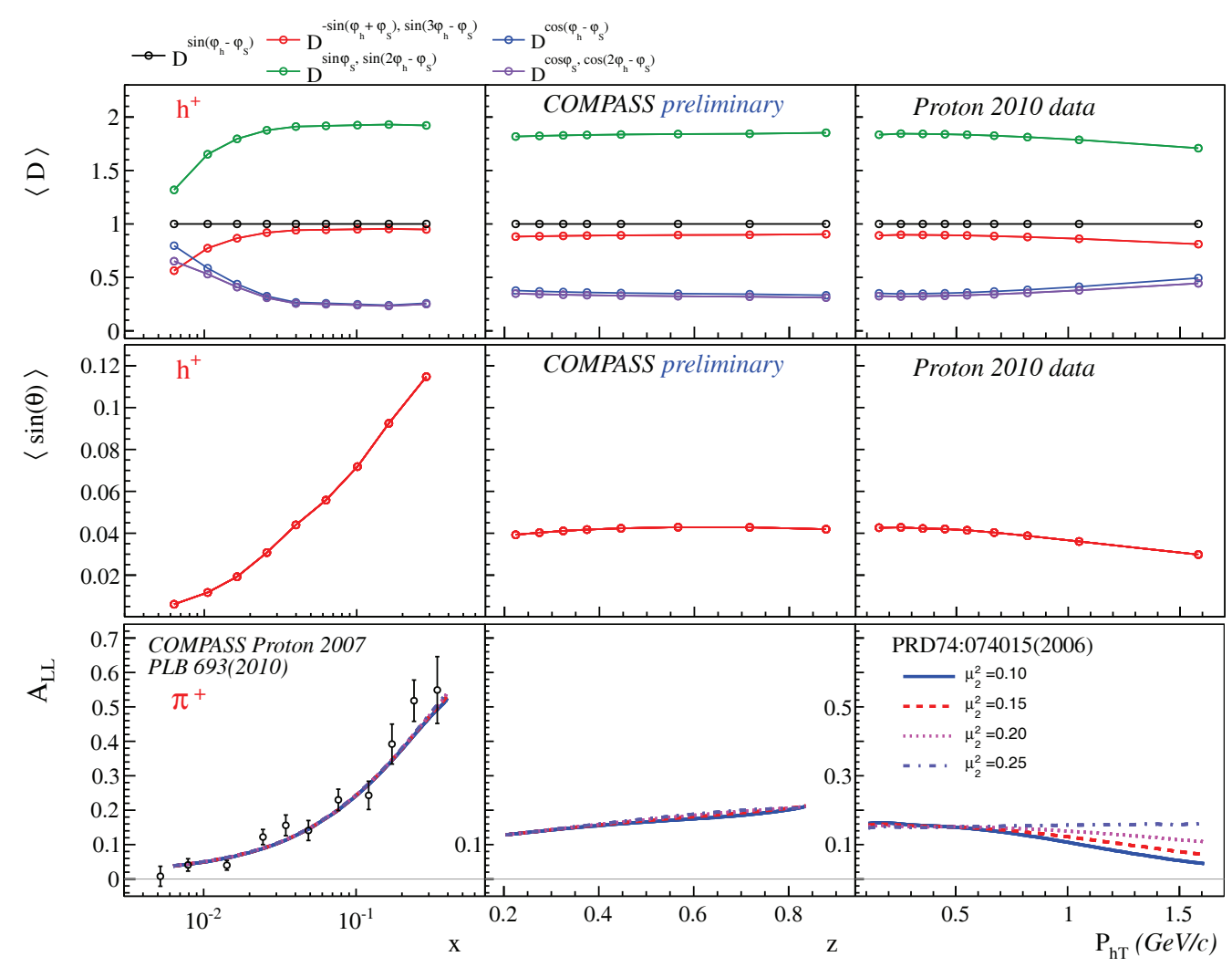

Figure 2: Mean depolarization factors $D$ (top), $\sin (\theta)$ factors (middle) and $A_{L L}$ asymmetry (bottom).

\section{Conclusions}

The preliminary results on six, additional to Collins and Sivers amplitudes, asymmetries from COMPASS proton 2010 data, have been presented. A non-zero trend has been observed for the $A_{L T}^{\cos \left(\phi_{h}-\phi_{s}\right)}$ and $A_{U T}^{\sin \left(\phi_{s}\right)}$ amplitudes, while the other four are found to be consistent with zero within the statistical accuracy. The measured kinematical dependencies of $A_{L T}^{\cos \left(\phi_{h}-\phi_{s}\right)}$ asymmetry are inline with the predictions given by several theoretical models. Combined with the previous COMPASS measurements and data from other experiments, these results give another possibility to access TMD PDFs and FFs, and to study the spin-structure of the nucleon. 

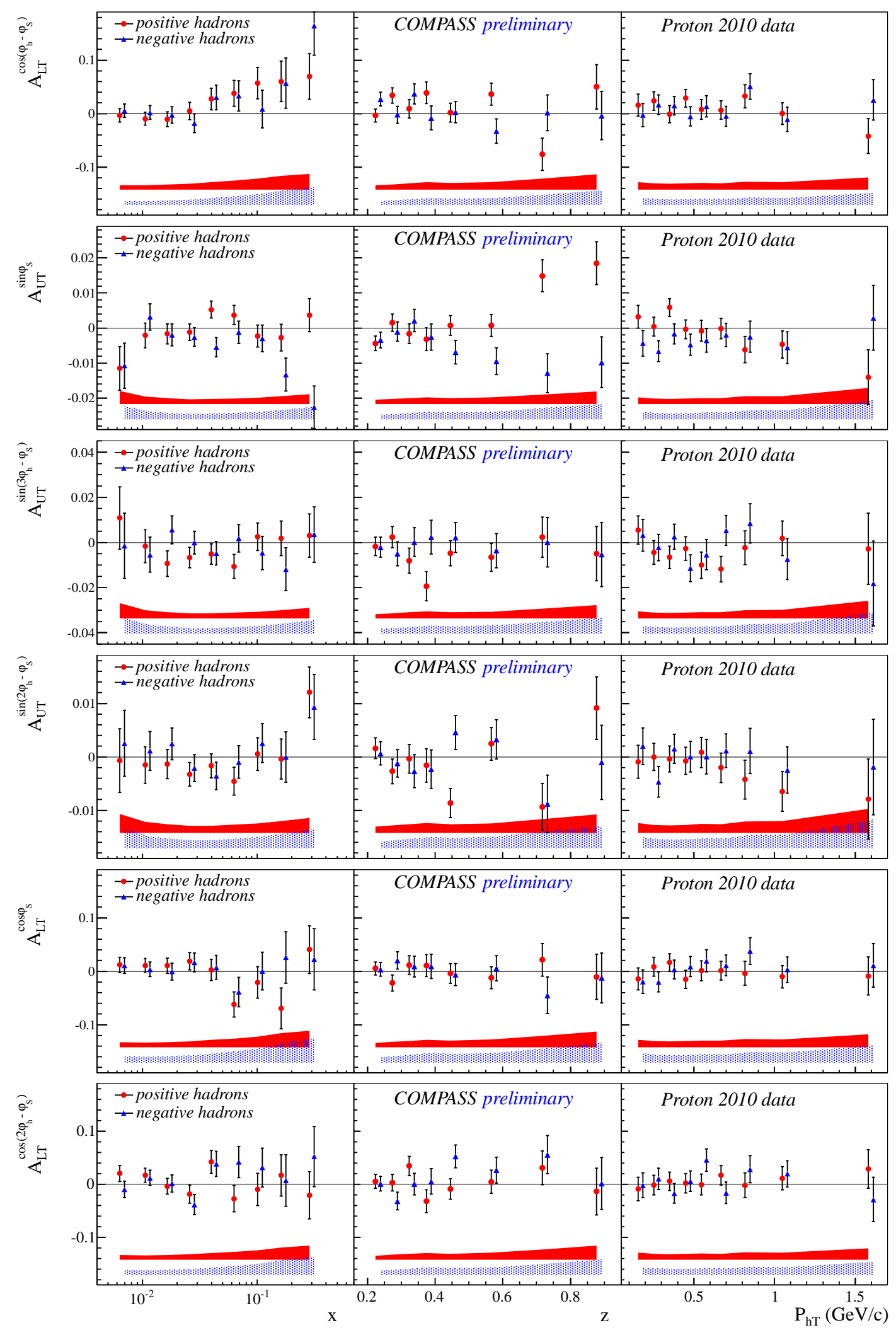

Figure 3: Six "Beyond Collins and Sivers" asymmetries at COMPASS (Proton 2010 data). 

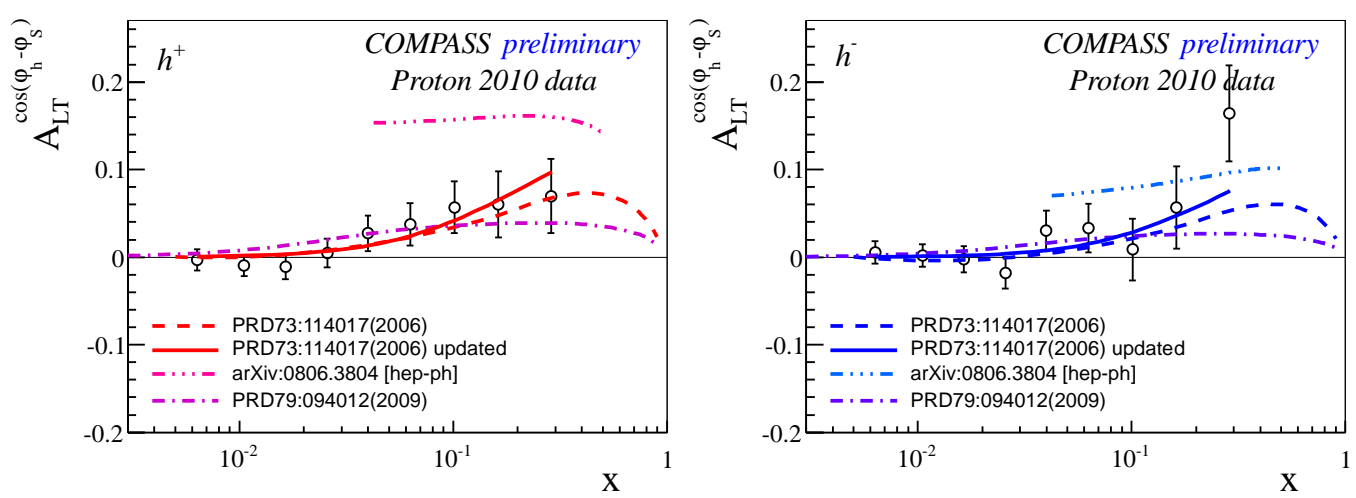

Figure 4: $A_{L T}^{\cos \left(\phi_{h}-\phi_{S}\right)}$ asymmetry: comparison with the theoretical predictions.

\section{References}

[1] P. Abbon et al. [COMPASS], Nucl. Instrum. Meth. A 577 (2007) 455, [arXiv:hep-ex/0703049].

[2] B. Parsamyan, Eur. Phys. J. ST 162, 89 (2008), [arXiv:0709 .3440] [hep-ex]].

[3] B. Parsamyan, J. Phys. Conf. Ser. 295(2011)012046, [arXiv: 1012.0155 [hep-ex]].

[4] B. Parsamyan, in proceedings of SPIN 2012 conference, [arXiv:1301.6615 [hep-ex]].

[5] A. Kotzinian, Nucl. Phys. B 441, 234 (1995), arXiv:hep-ph/9412283.

[6] A. Bacchetta et al., JHEP 0702, 093 (2007), [arXiv: hep-ph/0611265].

[7] M. Diehl and S. Sapeta, Eur. Phys. J. C 41 (2005) 515, [arXiv:hep-ph/0503023].

[8] M. G. Alekseev et al. [COMPASS], Phys. Lett. B 693 (2010) 227, [arXiv: 1007.406 ] [hep-ex]].

[9] M. Anselmino et al., Phys. Rev. D 74 (2006) 074015, [arXiv:hep-ph/0608048].

[10] M. G. Alekseev et al. [COMPASS], Eur. Phys. J. C 70 (2010) 39, [arXiv:1007.1562 [hep-ex]].

[11] P. J. Mulders and R. D. Tangerman, Nucl. Phys. B 461 (1996) 197, [arXiv:hep-ph/9510301].

[12] C. Adolph et al. [COMPASS], Phys. Lett. B 717 (2012) 376, [arXiv: 1205.512] [hep-ex]].

[13] C. Adolph et al. [COMPASS], Phys. Lett. B 717 (2012) 383, [arXiv:1205.5122 [hep-ex]].

[14] A. Kotzinian et al., Phys. Rev. D 73 (2006) 114017, [arXiv: hep-ph/0603194].

[15] S. Boffi et al., Phys. Rev. D 79, 094012 (2009), [arxiv:0903.127] [hep-ph]].

[16] A. Kotzinian, in proceedings of Transversity 2008 conference, [arXiv:0806.3804] [hep-ph]]. 